\title{
Efficacy of EMDR Therapy for Anxiety Disorders
}

\author{
Elisa Faretta \\ Mariella Dal Farra \\ Centro di Ricerca PIIEC e di Psicoterapia Integrata EMDR, Milan, Italy \\ CRSP Centro di Ricerca e Studi in Psicotraumatologia, Associazione EMDR Italia, Varedo, Italy
}

\begin{abstract}
Six randomized controlled trials (RCTs) investigated the efficacy of eye movement desensitization and reprocessing (EMDR) therapy for adults with anxiety disorders over a span of 20 years (1997-2017). Three RCTs focused on panic disorder, with or without agoraphobia (PDA); two studies targeted specific phobias, whereas the dependent variable of another RCT was "self-esteem," considered as a mediator factor for anxiety disorders. In four RCTs, EMDR therapy demonstrated a positive effect on panic and phobic symptoms, whereas one RCT on PDA was partly negative and one study failed in improving selfesteem in patients with anxiety disorders. Considered as a whole, these preliminary data suggest EMDR therapy may be effective not only for PD but also for specific phobias. Further controlled studies are needed to corroborate these findings and also to systematically evaluate the efficacy of EMDR therapy for generalized anxiety disorder, social anxiety, and agoraphobia. Because cognitive behavioral therapy (CBT) is presently considered a first-line treatment for anxiety disorders, controlled comparisons between EMDR therapy and CBT would be especially useful in future investigations of EMDR treatment of anxiety disorders.
\end{abstract}

Keywords: eye movement desensitization and reprocessing therapy; anxiety disorders; panic; randomized controlled trial; review

0 ver the last 22 years, six randomized controlled trials (RCTs) have investigated the efficacy of eye movement desensitization and reprocessing (EMDR) therapy for anxiety disorders in adults (see Table 1). EMDR is an evidence-based therapy for posttraumatic stress disorder (PTSD) and other trauma- and stress-related disorders, capable of addressing and substantially modifying disturbing memories of traumatic events. According to Shapiro's (2001) Adaptive Information Processing (AIP) model, stressful and/or traumatic experiences that are not adequately processed-especially if they occurred during the early years - can result in impaired coping skills and increased vulnerability to later stressful events. This, in turn, can lead to the development and maintenance of trauma- and stress-related disorders and other presenting problems such as anxiety disorders. EMDR's use in the treatment of anxiety disorders is based on the AIP-derived assumption that EMDR processing of related disturbing memories will reduce or eliminate the emotional, somatic, cognitive, and behavioral symptoms of anxiety.

This article is a brief narrative review with the purpose of examining EMDR therapy's efficacy for this clinical population. For the sake of brevity, the National Institute for Health and Care Excellence (NICE) guidelines for anxiety treatment are used to discuss recommended treatments. The reader should be cognizant that although other guidelines may make recommendations that differ from those of NICE, none recommend-or even mention-EMDR therapy for treatment of the anxiety disorders. 
The studies in this review were selected by searching the following databases: EBSCO Psychology and Behavioral Sciences Collection, Google Scholar, and the Francine Shapiro Library. Inclusion criteria for the review were RCTs, investigating EMDR treatment of adults with a diagnosed anxiety disorder. The review does not include studies that addressed presenting problems such as performance anxiety or healthrelated anxiety, as the participants in such studies were not diagnosed with an anxiety disorder, and it does not address the EMDR treatment of children with anxiety disorders.

\section{Generalized Anxiety Disorder}

The Diagnostic and Statistical Manual of Mental Disorders (DSM-5; American Psychiatric Association, 2013) defines generalized anxiety disorder (GAD) as "excessive anxiety and worry ... about a number of events or activities" with clinically significant distress or functional impairment (p. 222). The NICE (2019) clinical guideline for GAD recommends that if symptoms do not improve after education and active monitoring in primary care, patients should be provided cognitive behavioral therapy (CBT) or applied relaxation (or pharmacological treatment). CBT treatment for GAD focuses on exposure to anxiety, to create habituation and tolerance, and cognitive therapy to modify thoughts, that is, "cognitive biases, such as overgeneralization or maximization of danger" (Borza, 2017, p. 205).

GAD is a condition that tends to be linked to a plurality of variables, such as origin (more frequent in individuals with European origin than in individuals with non-European origin), gender (more females than men), and personality traits (e.g., "neuroticism"), but there is evidence that some cases "could be rooted in multiple disturbing experiences in a client's learning history" (cited in De Jongh \& Ten Broeke, 2009; Roemer, Molina, Litz, \& Borkovec, 1997). This observation provides the rationale for the application of EMDR therapy to GAD, which addresses memories of such disturbing experiences.

At the present time, there are no RCTs that investigated EMDR treatment for GAD, but two preliminary studies were carried out (Farima, Dowlatabadi, \& Behzadi, 2015; Gauvreau \& Bouchard, 2008). Both studies targeted experiential contributors to GAD and the current and anticipated situations that caused excessive worry, and resulted in a reduction of anxiety scores that was maintained at short-term follow-up.

No claim of efficacy can be made on the basis of these preliminary results, but the data are interesting enough to foster the implementation of RCTs on EMDR treatment for GAD.

\section{Social Anxiety Disorder}

The DSM- 5 states that social anxiety disorder (SAD) is characterized by "marked, or intense, fear or anxiety of social situations in which the individual may be scrutinized by others" (APA, 2013, p. 203). According to NICE (2013), treatment options indicated for adults with SAD are CBT models specifically developed to treat the disorder, which may provide strategies such as experiential exercises and systematic training, examination and modification of core beliefs (Clark \& Wells, 1995), graduated social exposure, and cognitive restructuring (Heimberg, 1995). If the patient declines CBT and pharmacological interventions, short-term psychodynamic psychotherapy can be considered. The contribution of EMDR therapy for SAD lies in the possibility of resolving memories of stressful social events that possibly played a role in the development of the disorder and/or in its maintenance (i.e., working as a trigger for similar events).

There are no controlled trials on the efficacy of EMDR for SAD. One single case report (Sun \& Chiu, 2006) concerned the intervention with a psychiatric outpatient with long-term social phobia who was successfully treated with EMDR combined with mindfulness meditation.

Although sports anxiety, performance anxiety, and test anxiety can be considered forms of SAD, none of the controlled trials or case studies investigating EMDR's efficacy for these presentations involved participants with a formal diagnosis. Four RTCs were carried out to investigate EMDR's treatment of test anxiety, and all produced significant and rapid reductions in presenting symptoms (Cook-Vienot $\&$ Taylor, 2012; Enright, Baldo, \& Wykes, 2000; Maxfield \& Melnyk, 2000; Stevens \& Florell, 1999). There appears to be solid and consistent evidence for EMDR's use for individuals with test anxiety.

Many case studies have looked at EMDR's use for performance anxiety, public speaking anxiety, and sports anxiety. It is clear that EMDR therapy has potential in this area, but the research is very heterogeneous, and systematic studies are needed to evaluate EMDR's efficacy before any conclusions can be made.

\section{Specific Phobias}

According to the DSM-5, individuals with specific phobia experience marked fear that is elicited only by the presence of a particular situation or object (APA, 2013, 
p. 198). Recommended treatment typically involves CBT techniques of exposure and response prevention, either through in vivo exposure or virtual reality. EMDR treatment of specific phobias is based on the hypotheses that experiences of intense, emotional disruption related to specific situations (e.g., feeling helpless in the dentist's office) can precipitate the development of specific phobias (Oosterink, De Jongh, \& Aartman, 2009), and that processing such memories with EMDR can decrease the intensity and pervasiveness of related phobia symptoms. Two controlled case studies described EMDR's successful treatment of traumatically induced dental phobia (De Jongh, Van den Oord, \& Ten Broeke, 2002) and claustrophobia (Lohr, Tolin, \& Kleinknecht, 1996).

A large non-randomized study $(N=184)$ found that trauma-focused CBT (TF-CBT) and EMDR, both combined with in vivo exposure treatment, were effective for diagnosed travel phobia ( $57 \%$ of the sample), or milder travel anxiety arising as a result of a road traffic accident (De Jongh, Holmshaw, Carswell, \& Van Wijk, 2011). Following an average course of 7.3 sessions, participants in both treatment conditions showed equally large, and clinically significant, decreases in symptoms on the Impact of Event Scale, Hospital Anxiety and Depression Scale (HADS), and General Health Questionnaire, therapist ratings of treatment outcome, and a return to driving or travelling by car or motor-bike. Unfortunately, the lack of randomization limits any kind of conclusion that can be drawn.

Two RCTs have investigated EMDR treatment of participants diagnosed with specific phobia See Table 1. In the Doering, Ohlmeier, De Jongh, Hofmann, and Bisping (2013) study, 31 medication-free patients were randomly assigned to either a wait-list control condition or three EMDR sessions (90 minutes each) that targeted memories of negative dental events. Dental anxiety was assessed using the Dental Anxiety Questionnaire, the Dental Fear Survey, a behavior test, and dental attendance at 1-year followup. EMDR was associated with significant reductions of dental anxiety and avoidance behavior: these effects were still significant at 3 months and 12 months after treatment. After 1 year, $83.3 \%$ of the EMDR patients were in regular dental treatment.

Triscari, Faraci, Catalisano, D'Angelo, and Urso (2015) investigated the treatment of flight anxiety by randomly assigning 65 self-referred participants diagnosed with specific phobia to one of three conditions: CBT integrated with systematic desensitization, CBT combined with EMDR, and CBT combined with virtual reality exposure therapy (VRET). All conditions provided 10 weekly sessions of 2 hours each. The first three sessions provided group treatment consisting of psychoeducation, CBT and relaxation techniques, and education about flying. Sessions 4-6 were specific to each treatment group. Sessions 7-10 provided group treatment: visiting the air traffic control tower, asking questions to a pilot and an air traffic controller, experiencing simulated flights, and then a real flight. There were significant decreases for all groups on the Flight Anxiety Situations Questionnaire and Flight Anxiety Modality Questionnaire with results maintained at 1-year follow-up, and no difference between treatments. Findings suggest that combining CBT with either EMDR or VRET seemed as efficient as traditional CBT integrated with systematic desensitization.

Together with panic disorder (PD), panic disorder with or without agoraphobia (PDA), specific phobia is the most investigated anxiety disorder regarding the efficacy of EMDR therapy. Two RCTs have been carried out respectively on dental phobia (Doering et al., 2013) and flight anxiety (Triscari et al., 2015), both with significative results, which were maintained at 1-year follow-ups.

These positive outcomes are corroborated by one large, non-randomized study on travel anxiety (De Jongh et al., 2011) and two controlled case studies on dental phobia (De Jongh et al., 2002) and claustrophobia (Lohr et al., 1996).

\section{Panic Disorder, With/Without Agoraphobia}

PD is characterized by "recurrent unexpected panic attacks" (APA, 2013, p. 208) and agoraphobia is defined as "marked fear or anxiety" in situations where "escape might be difficult or help might not be available" for panic-like symptoms (APA, 2013, p. 217). According to NICE (2019), evidence-based treatment options for PD in adults are CBT, medication, and self-help. The CBT model views panic attacks as originating from an anxious interpretation of somatic symptoms that are understood as a prelude to some catastrophic, physical event (e.g., tachycardia as a symptom of imminent heart attack). Coherently, CBT addresses the cognitive bias with cognitive restructuring techniques and deconditioning (Faretta $\&$ Leeds, 2017).

The AIP model proposes a different theoretical framework: it hypothesizes that early stressful experiences can facilitate a resort to dissociative defence mechanisms, especially cognitive-affective dissociation, undermining the coping skills and facilitating the development of PD. Furthermore, panic represents a potentially traumatic experience per se because 
TABLE 1. Randomized Controlled Trials That Have Investigated the Efficacy of EMDR Therapy for Anxiety Disorders in Adults

\begin{tabular}{|c|c|c|c|c|c|c|}
\hline Author (Year) & Diagnosis & $N$ & $\begin{array}{l}\text { Control } \\
\text { Condition }\end{array}$ & $\begin{array}{l}\text { Number } \\
\text { of } \\
\text { Sessions }\end{array}$ & $\begin{array}{l}\text { Standard } \\
\text { Protocol } \\
\text { / Variations }\end{array}$ & Findings \\
\hline $\begin{array}{l}\text { Feske \& Goldstein } \\
\text { (1997) }\end{array}$ & $\begin{array}{l}\text { Panic disorder } \\
\text { with } \\
\text { agoraphobia } \\
(D S M-I V-T R)\end{array}$ & 43 & $\begin{array}{l}\text { WL or } \\
\text { EFER }\end{array}$ & 6 & EMDR & $\begin{array}{l}\text { EMDR }>\text { WL; EMDR }>\text { EFER on } \\
\text { two of five primary measures. Not } \\
\text { significant at 3-month follow-up. }\end{array}$ \\
\hline $\begin{array}{l}\text { Goldstein et al. } \\
(2000)\end{array}$ & $\begin{array}{l}\text { Panic disorder } \\
\text { with } \\
\text { agoraphobia } \\
(D S M-I V-T R)\end{array}$ & 46 & $\begin{array}{l}\text { WL or } \\
\text { ART }\end{array}$ & 6 & EMDR & $\begin{array}{l}\text { EMDR }>\text { WL on } \\
\text { panic/ agoraphobia severity but } \\
\text { not on frequency of panic attacks. } \\
\text { EMDR = ART. Stable at 1-month } \\
\text { follow-up. }\end{array}$ \\
\hline Horst et al. (2017) & $\begin{array}{l}\text { Panic disorder } \\
(D S M-5)\end{array}$ & 84 & $\mathrm{CBT}$ & 13 & $\begin{array}{l}\text { EMDR (audio } \\
\text { tones) }\end{array}$ & $\begin{array}{l}\text { EMDR = CBT on ACQ, BSQ and } \\
\text { QOL while MI was inconclusive. } \\
\text { Significant at 3-month follow-up. }\end{array}$ \\
\hline $\begin{array}{l}\text { Doering et al. } \\
(2013)\end{array}$ & $\begin{array}{l}\text { Dental phobia } \\
\text { (DSM-IV-TR) }\end{array}$ & 31 & WL & 3 & EMDR & $\begin{array}{l}\text { EMDR }>W L \text { for reductions of } \\
\text { dental anxiety and avoidance } \\
\text { behavior. Significant at } 3 \text { - and } \\
\text { 12-month follow-up. }\end{array}$ \\
\hline Triscari et al. (2015) & $\begin{array}{l}\text { Flying anxiety } \\
(D S M-5)\end{array}$ & 65 & $\begin{array}{l}\text { CBT-SD or } \\
\text { CBT- } \\
\text { VRET }\end{array}$ & 10 & CBT+EMDR & $\begin{array}{l}\mathrm{CBT}+\mathrm{EMDR}=\mathrm{CBT}+\mathrm{SD}= \\
\mathrm{CBT}+\mathrm{VRET} \text { for decrease of } \\
\text { flight anxiety. Significant at 1-year } \\
\text { follow-up. }\end{array}$ \\
\hline Staring et al. (2016) & $\begin{array}{l}\text { Anxiety } \\
\text { disorders } \\
(D S M-5)\end{array}$ & 47 & COMET & 6 & EMDR & $\begin{array}{l}\text { EMDR < COMET in improving } \\
\text { self-esteem. EMDR reduces } \\
\text { COMET effects but not vice versa. }\end{array}$ \\
\hline
\end{tabular}

Note. ACQ $=$ Agoraphobic Cognitions Questionnaire; ART $=$ Association and Relaxation Therapy; BSQ $=$ Body Sensations Questionnaire; $\mathrm{CBT}=$ cognitive behavioral therapy; $\mathrm{CBT}+\mathrm{EMDR}=\mathrm{CBT}$ combined with EMDR; CBT + VRET = CBT combined with VRET; COMET = Competitive Memory Training; EFER = Eye Fixation Exposure and Reprocessing; CBT + SD = CBT combined with systematic desensitization; DSM = Diagnostic and Statistical Manual of Mental Disorders; MI = Mobility Inventory; QOL= quality of life; VRET $=$ virtual reality exposure therapy.

$>$ indicates significantly better than; <indicates significantly worse than.

of the intense fear and feeling of helplessness that defines it (Faretta, 2018). It has been theorized (Faretta, 2013; Fernandez \& Faretta, 2007; Leeds, 2012) that EMDR therapy can be a useful approach to PD in order to address memories of panic attacks (the first, the worst, and the last), current triggers that can elicit panic, and early contributory experiences (e.g., disorganized attachment, adverse childhood experiences).

EMDR therapy's efficacy for PD has been investigated with many case studies (single and series), one controlled non-randomized trial, and three RCTs. A qualitative analysis of RCTs that tested EMDR's impact on PD showed that the study with better results (Horst et al., 2017) used a different treatment plan than the studies with less positive outcomes
(Feske \& Goldstein, 1997; Goldstein, De Beurs, Chambless, \& Wilson, 2000). This suggests the possibility that efficacy and stability of the results may depend on the choice and the sequencing of targets. Specifically, in the Horst et al. study (2017), case conceptualization for the EMDR group was formulated according to De Jongh, Ten Broeke, and Meijer "First method" of the "Two Method Approach" (2010) that "deals with symptoms whereby memories of the etiological and/or aggravating events were meaningfully specified on a time line [...]. The memories that evoked the most disturbance, e.g. the first or worst panic attack, were reprocessed first [...]. Subsequently, other memories that were considered to contribute to a patient's current symptoms were targeted in the same way" (Horst et al., 2017, pp. 3-4). 
The results of numerous case studies (Bhagwagar, 2016; Fernandez \& Faretta, 2007; Goldstein, 1995; Goldstein \& Feske, 1994; Leeds, 2009, 2016; Nadler, 1996) tend to show consistent positive outcomes. The researchers applied different treatment protocols, such as Shapiro's (2001) EMDR phobia protocol, De Jongh et al. (2010) "Two Method Approach," and Leeds (2012) Models I and II treatment plans.

Faretta's (2013) non-randomized controlled study provided 12 sessions of EMDR or 12 sessions of CBT to 19 participants who met DSM-IV-TR criteria for PD with or without agoraphobia; cases of complex PD were excluded. The EMDR intervention followed the standard protocol developed by Shapiro $(1999,2001)$, tailored by Faretta for the treatment of PD in accordance with the case reports of Fernandez and Faretta (2007) and Leeds (2009). Coherently, special attention was paid to the preparation phase, treatment plan (sequencing of the selected targets), current triggers, and future templates. Results indicate that both 12 sessions of EMDR or 12 sessions of CBT were effective in the treatment of PD. The outcomes were stable at 1-year follow-up. A repeated measure analysis of variance (ANOVA) pointed out significant "Time $\times$ Treatment" interaction for frequency of panic attacks in the EMDR group, tracking "a continuing decrease in frequency of panic attacks for participants with PD or PDA in the EMDR condition at follow-up that was significantly greater than that found in the CBT treatment group" (Faretta, 2013, p. 131).

The first RCT was carried out by Feske and Goldstein (1997) (see Table 1). In addition to investigating EMDR treatment of PD, the study also evaluated the role of eye movements by comparing EMDR-witheye-movements to EMDR-with-eyes-fixed, eye fixation exposure and reprocessing (EFER). They assigned 43 outpatients (including 2 with PDA) to six sessions of EMDR, six sessions of EFER, or waiting list. A comparison of EMDR with waiting list showed that EMDR was significantly superior in reducing panic and panicrelated symptoms. Compared with EFER, EMDR was more effective on two of five primary measures at post-test, but the difference was not maintained at 3-month follow-up, suggesting that the eye movement component might not be necessary.

The second RCT was published by Goldstein et al. (2000). The sample consisted of 46 PDA patients (DSM-IV-TR) who were subdivided into three groups: the first received six EMDR sessions; the second, six association and relaxation therapy (ART) sessions (a placebo); the third, waiting list. "Throughout treatment, therapists in both conditions were prohibited from using interventions outside the realm of the protocol such as anxiety management training, cognitive restructuring, in vivo exposure, and exploration of intrapsychic issues" (Goldstein et al., 2000, p. 949). EMDR's results were significantly superior to wait list in decreasing the severity of panic/agoraphobia but not the number of panic attacks. Furthermore, no significant differences between EMDR and ART groups emerged and EMDR patients "fared no better than those in the attention-placebo group" (2000, p. 953). These negative results were stable at 1-month follow-up.

A third, more recent study on EMDR and PD contradicted these findings: Horst et al. (2017) carried out a comparative RCT contrasting 13 sessions of EMDR with 13 sessions of CBT on a sample of 84 patients with PD (DSM-5). The EMDR treatment used Shapiro's (2001) standard EMDR protocol. Targets were memories of disturbing events identified using the "first method" of the two-method approach (De Jongh et al., 2010). The results indicate non-inferiority of EMDR to CBT on two out of three primary measures (Agoraphobic Cognitions Questionnaire [ACQ], and Body Sensations Questionnaire [BSQ]), whereas the third variable (Mobility Inventory [MI]) was inconclusive. The results remained significant at 3-month follow-up. A limitation of this research was the use of audio tones: "This implies that when eye movements would have been applied in the present study the results might have been more profound" (Horst et al., 2017, p. 7).

The findings of the Feske and Goldstein (1997) and Goldstein et al. (2000) RCTs contrast with the positive outcomes of RCT by Horst et al. and with the stability of results in the EMDR condition over time found in Faretta's (2013) non-randomized, controlled study. A possible explanation deals with the development of the treatment plan. Nadler (1996), Shapiro and Forrest (1997), Fernandez and Faretta (2007), Leeds (2009, 2012, 2016), and Bhagwagar (2016) recommended that EMDR therapy for PD include the reprocessing of early contributory experiences, as well as desensitization of later etiological factors. Special attention should also be accorded to the preparation phase and to the implementation of future templates.

\section{Low Self-Esteem in Patients With Diagnosed Anxiety Disorder}

Shapiro's (2001) AIP model views symptoms such as low self-esteem as arising from related disturbing 
memories and predicts that processing these memories will ameliorate symptoms of anxiety and depression. This assumption was tested in a study by Staring et al. (2016), who carried out a randomized controlled crossover trial of EMDR versus Competitive Memory Training (COMET) in 47 patients with low self-esteem and a primary anxiety disorder $(51 \%$ with diagnosed PTSD) (see Table 1).

Group $1(N=24)$ received six EMDR sessions first and then six COMET sessions; group $2(N=23)$ vice versa. EMDR targeted negative memory representations that were proposed to maintain low self-esteem, while COMET focused on positive representations for enhancing self-esteem and provided daily homework. Assessments were made at baseline (T0), end of first treatment (T1), and end of second treatment (T2). COMET was significantly more effective in improving self-esteem than EMDR therapy (effect sizes of 1.25 vs. 0.46 , respectively). Another finding was that EMDR treatment reduced the effectiveness of subsequent COMET. Authors concluded by stating that enhancing positive representations seemed to be more effective than desensitizing negative ones to improve self-esteem. They also noted that the EMDR participants may have experienced exacerbation of anxiety symptoms, such as PTSD flashbacks, as the PTSDrelated traumatic memories were not treated in the study.

\section{Discussion}

In summary, EMDR therapy has demonstrated in four RCTs a positive effect on panic and phobic symptoms (Doering et al., 2013; Feske \& Goldstein, 1997; Horst et al., 2017; Triscari et al., 2015), whereas one RCT on PDA was in part negative (Goldstein et al., 2000) and one study failed in improving selfesteem in patients with anxiety disorders (Staring et al., 2016). It should be noted that the studies with poorer outcomes did not address intra-psychic issues or traumatic memories.

These preliminary data suggest EMDR therapy may be effective both for PD and specific phobias, prompting the opportunity to pursue further RCTs on the topics. The systematic evaluation of EMDR's efficacy for GAD, SAD, agoraphobia, and other specific phobias is equally needed. Considering that CBT currently represents the first-line treatment for anxiety disorders, controlled comparisons between EMDR therapy and CBT would be especially relevant.

\section{Clinical Implications}

Shapiro's AIP model provides an innovative approach to the treatment of anxiety disorders, targeting the traumatic aetiology that often contributes to the development and/or maintenance of the condition. The RCTs and the controlled case studies carried out to date are too exiguous to draw conclusions, but, taken as a whole, they seem to point out the cruciality of AIP model's "three-pronged approach." Working on the past (elaboration of the contributory experiences), the present (decrease of the hyperarousal elicited by current triggers), and the future (relapse prevention with flash-forwards and future templates) is a key factor to gain steady outcomes with EMDR therapy. The three-pronged approach applied to anxiety disorders does not require a protracted time but calls for a calibrated treatment plan. Coherently, EMDR therapy can be usefully applied after a careful, clinical evaluation of the role played by stressful or traumatic events in the case history of the patient. While specific EMDR protocols have been developed to address PD and PD and agoraphobia (Leeds, 2012), PD(Faretta, 2018), and specific phobias (Shapiro, 2001), these modified procedures are in need of research evaluation to determine their effectiveness.

\section{References}

American Psychiatric Association. (2013). Diagnostic and statistical manual of mental disorders (5th ed.). Washington, DC: Author.

Bhagwagar, H. (2016). EMDR in the treatment of panic disorder with agoraphobia: A case description. Journal of EMDR Practice and Research, 10(4), 256-274. doi:10.1891/1933-3196.10.4.256

Borza, L. (2017). Cognitive-behavioral therapy for generalized anxiety. Dialogues in Clinical Neuroscience, 19(2), 203208.

Clark, D. M., \& Wells, A. (1995). A cognitive model of social phobia. Social Phobia: Diagnosis, Assessment, and Treatment, 41(68), 00022-00023.

Cook-Vienot, R., \& Taylor, R. J. (2012). Comparison of eye movement desensitization and reprocessing and biofeedback/stress inoculation training in treating test anxiety. Journal of EMDR Practice and Research, 6(2), 62-72. doi:10.1891/1933-3196.6.2.62

De Jongh, A., Holmshaw, M., Carswell, W., \& Van Wijk, A. (2011). Usefulness of a trauma-focused treatment approach for travel phobia. Clinical Psychology \& Psychotherapy, 18(2), 124-137. doi:10.1002/cpp.680

De Jongh, A., \& Ten Broeke, E. (2009). EMDR and the anxiety disorders: Exploring the current status. 
Journal of EMDR Practice and Research, 3(3), 133-140. doi:10.1891/1933-3196.3.3.133

De Jongh, A., Ten Broeke, E., \& Meijer, S. (2010). Two method approach: A case conceptualization model in the context of EMDR. Journal of EMDR Practice and Research, 4, 12-21. doi:10.1891/1933-3196.4.1.12

De Jongh, A., Van den Oord, H. J. M., \& Ten Broeke, E. (2002). Efficacy of eye movement desensitization and reprocessing in the treatment of specific phobias: Four single-case studies on dental phobia. Journal of Clinical Psychology, 58(12), 1489-1503. doi:10.1002/jclp.10100

Doering, S., Ohlmeier, M. C., De Jongh, A., Hofmann, A., \& Bisping, V. (2013). Efficacy of a trauma-focused treatment approach for dental phobia: A randomized clinical trial. European Journal of Oral Sciences, 121(6), 584-593. doi:10.1111/eos. 12090

Enright, M., Baldo, T. D., \& Wykes, S. D. (2000). The efficacy of eye movement desensitization and reprocessing therapy technique in the treatment of test anxiety of college students. Journal of College Counseling, 3(1), 36-48. doi:10.1002/j.2161-1882.2000.tb00162.x

Faretta, E. (2013). EMDR and cognitive behavioral therapy in the treatment of panic disorder: A comparison. Journal of EMDR Practice and Research, 7(3), 121-133. doi:10.1708/1071.11735

Faretta, E. (2018, October). EMDR e disturbo di panico: Dalle teorie integrate al modello di intervento nella pratica. Edra, Milano.

Faretta, E., \& Leeds, A. (2017). EMDR therapy of panic disorder and agoraphobia: A review of the existing literature. Clinical Neuropsychiatry, 14, 5, 330-340.

Farima, R., Dowlatabadi, S., \& Behzadi, S. (2015). The effectiveness of eye movement desensitization and reprocessing (EMDR) in reducing pathological worry in patients with generalized anxiety disorder: A preliminary study. Archives of Psychiatry and Psychotherapy, 1, 3343. doi:10.12740/APP/39259

Fernandez, I., \& Faretta, E. (2007). Eye movement desensitization and reprocessing in the treatment of panic disorder with agoraphobia. Clinical Case Studies, 6(1), 44-63. doi: $10.1177 / 1534650105277220$

Feske, U., \& Goldstein, A. J. (1997). Eye movement desensitization and reprocessing treatment for panic disorder: A controlled outcome and partial dismantling study. Journal of Consulting and Clinical Psychology, 65(6), 1026. doi:10.1037 / / 0022-006x.65.6.1026

Gauvreau, P., \& Bouchard, S. (2008). Preliminary evidence for the efficacy of EMDR in treating generalized anxiety disorder. Journal of EMDR Practice and Research, 2(1), 2640. doi:10.1891/1933-3196.2.1.26

Goldstein, A. (1995). Treatment of panic disorder with agoraphobia: Going beyond the barrier. In Session: Psychotherapy in Practice, 1(3), 83-98.

Goldstein, A. J., De Beurs, E., Chambless, D. L., \& Wilson, K. A. (2000). EMDR for panic disorder with agoraphobia: Comparison with waiting list and credible attentionplacebo control conditions. Journal of Consulting and
Clinical Psychology, 68(6), 947. doi:10.1016/08876185(94)00023-9

Goldstein, A. J., \& Feske, U. (1994). Eye movement desensitization and reprocessing for panic disorder: A case series. Journal of Anxiety Disorders, 8(4), 351-362. doi:10.1016/0887-6185(94)00023-9

Heimberg, R. G., Liebowitz, M. R., Hope, D. A., Schneier, F. R. (1995). Social phobia: Diagnosis, assessment, and treatment. New York: Guilford Press.

Horst, F., Den Oudsten, B., Zijlstra, W., de Jongh, A., Lobbestael, J., \& De Vries, J. (2017). Cognitive behavioral therapy vs. eye movement desensitization and reprocessing for treating panic disorder: A randomized controlled trial. Frontiers in Psychology, 8, 1409. doi:10.3389/fpsyg.2017.01409

Leeds, A. M. (2009). A guide to the standard EMDR protocols for clinicians, supervisors, and consultants. New York, NY: Springer Publishing.

Leeds, A. M. (2012). EMDR treatment of panic disorder and agoraphobia: Two model treatment plans. Journal of EMDR Practice and Research, 6(3), 110-119. doi:10.1891/1933-3196.6.3.110

Leeds, A. M. (2016). A guide to the standard EMDR therapy protocols for clinicians, supervisors, and consultants (2nd ed.). New York, NY: Springer Publishing.

Lohr, J. M., Tolin, D. F., \& Kleinknecht, R. A. (1996). An intensive design investigation of eye movement desensitization and reprocessing of claustrophobia. Journal of Anxiety Disorders, 10(1), 73-88. doi:10.1016/08876185(95)00036-4

Maxfield, L., \& Melnyk, W. T. (2000). Single session treatment of test anxiety with eye movement desensitization and reprocessing (EMDR. International Journal of Stress Management, 7(2), 87-101. doi:10.1023/A:1009580101287

Nadler, W. (1996). EMDR: Rapid treatment of panic disorder. International Journal of Psychiatry, 2, 1-8.

National Institute for Health and Care Excellence. (2013). Social anxiety disorder: Recognition, assessment and treatment. Clinical guideline [CG159]. Retrieved from https: / / www.nice.org.uk/guidance/ cg159

National Institute for Health and Care Excellence. (2019). Generalised anxiety disorder and panic disorder in adults: Management. Clinical guideline [CG113]. Retrieved from https: / / www.nice.org.uk/guidance / cg113

Oosterink, F. M. D., De Jongh, A., \& Aartman, I. H. A. (2009). Negative events and their potential risk of precipitating pathological forms of dental anxiety. Journal of Anxiety Disorders, 23(4), 451-457. doi:10.1016/j.janxdis.2008.09.002

Roemer, L., Molina, S., Litz, B.T., \& Borkovec, T.D. (1997). Preliminary investigation of the role of previousexposure to potentially traumatizing events ingeneralized anxiety disorder.. Depression and Anxiety, 4, 134-138. doi:10.1002/(SICI)1520-6394(1996)4:3 <134::AID-DA6> 3.0.CO;2-G

Shapiro, F. (1999). Eye movement desensitization and reprocessing (EMDR) and the anxiety disorders: Clinical 
and research implications of an integrated psychotherapy treatment. Journal of Anxiety Disorders, 13(1-2), 35-67.

Shapiro, F. (2001). Eye movement desensitization and reprocessing (EMDR): Basic principles, protocols, and procedures. New York: Guilford Press.

Shapiro, F., \& Forrest, M. S. (1997). EMDR: The breakthrough therapy for overcoming anxiety, stress, and trauma. New York: Basic Books.

Staring, A. B. P., van den Berg, D. P. G., Cath, D. C., Schoorl, M., Engelhard, I. M., \& Korrelboom, C. W. (2016). Self-esteem treatment in anxiety: A randomized controlled crossover trial of eye movement desensitization and reprocessing (EMDR) versus competitive memory training (COMET) in patients with anxiety disorders. Behaviour Research and Therapy, 82, 11-20. doi:10.1016/j.brat.2016.04.002

Stevens, M. J., \& Florell, D. W. (1999). EMDR as a treatment for test anxiety. Imagination, Cognition and Personality, 18(4), 285-296. doi:10.2190/FJWQ-HKQQ-UEJW6VLH

Sun, T. F., \& Chiu, N. (2006). Synergism between mindfulness meditation training, and eye movement desensitization and reprocessing in psychotherapy of social phobia. Chang Gung Medical Journal, 29(4), 1-4.
Triscari, M. T., Faraci, P., Catalisano, D., D’Angelo, V., \& Urso, V. (2015). Effectiveness of cognitive behavioral therapy integrated with systematic desensitization, cognitive behavioral therapy combined with eye movement desensitization and reprocessing therapy, and cognitive behavioral therapy combined with virtual reality exposure therapy methods in the treatment of flight anxiety: A randomized trial. Neuropsychiatric Disease and Treatment, 11, 2591. doi:10.2147/NDT.S93401

Disclosure. Elisa Faretta receives income from published books on EMDR therapy, for the training of licensed professionals in EMDR therapy, and as a speaker at conferences and conventions. Mariella Dal Farra has no affiliations with any commercial interests related to the subjects discussed within this article.

Correspondence regarding this article should be directed to Mariella Dal Farra, Via San Gregorio nº 4, 20124 Milan (Mi), Italy. E-mail: m.dalfarra@fastwebnet.it 